\title{
Sitting and chronic disease: where do we go from here?
}

\author{
Katrien Wijndaele ${ }^{1} \cdot$ Genevieve N. Healy $^{2,3,4}$
}

Received: 8 January 2016 / Accepted: 22 January 2016/Published online: 5 February 2016

(C) Springer-Verlag Berlin Heidelberg 2016

Keywords Activity · Cardiometabolic · Cardiovascular . Chronic disease $\cdot$ Diabetes $\cdot$ Objective $\cdot$ Patterns $\cdot$ Posture . Public health $\cdot$ Sitting

In this issue of Diabetologia, van der Berg et al report the results of an interesting observational study [1]. The authors examined the cross-sectional associations of objectively measured sitting with type 2 diabetes and cardiometabolic risk in a sample of nearly 2,500 middle-aged Dutch adults enriched with type 2 diabetes patients. This study is an important addition to the literature aiming to examine the health consequences of sedentary behaviour, recently defined as any waking activity with low energy expenditure and performed in a sitting or reclining posture [2]. The most important asset of the work by van der Berg et al [1] is the implementation, in a large participant sample, of a thigh-worn activity monitor that accurately discriminates between sitting/lying and non-sitting/ lying postures. Using this methodology the authors were able to relate total sitting time and indicators of sitting patterns (i.e. the manner in which sitting time is accumulated) to glucose metabolism status and prevalence of criteria of the metabolic

Katrien Wijndaele

katrien.wijndaele@mrc-epid.cam.ac.uk

1 MRC Epidemiology Unit, Institute of Metabolic Science, University of Cambridge, Box 285, Addenbrooke's Hospital, Hills Road, Cambridge CB2 0QQ, UK

2 School of Public Health, The University of Queensland, Brisbane, QLD, Australia

3 Baker IDI Heart and Diabetes Institute, Melbourne, VIC, Australia

4 School of Physiotherapy, Curtin University, Perth, WA, Australia syndrome. They found that each additional hour daily of sitting time was significantly associated with increased odds of having type 2 diabetes $(1.22,95 \%$ CI $1.13,1.32)$ and the metabolic syndrome $(1.39,95 \%$ CI $1.27,1.53)$. This finding was irrespective of time spent in higher intensity activity and a wide range of socio-demographic, behavioural and healthrelated confounding factors. The accumulation patterns of this sitting time, whether examined as number of interruptions in sitting, number of prolonged (i.e. $\geq 30 \mathrm{~min}$ ) sitting bouts or average sitting bout duration, however, showed much weaker associations.

\section{Clinical and public health relevance of sedentary behaviour}

Interest in sitting as a risk factor for type 2 diabetes was sparked 15 years ago by $\mathrm{Hu}$ et al $[3,4]$. They were the first to associate high levels of TV viewing and other types of sedentary behaviour with increased risk for incident type 2 diabetes. As highlighted in a fairly recent meta-analysis in this journal [5], the link between sedentary behaviour and type 2 diabetes is one of the most consistently observed in this field - a field that has experienced an exponential rise in scientific, media and general community interest. This increased interest has also resulted in the emergence of preliminary public health guidelines focusing on reductions in prolonged sitting (for example, see [6]).

Sedentary behaviour is, however, still a fairly young research area, both in terms of advancements in measurement methodology as well as establishment of robust observational longitudinal and experimental evidence. Current metaanalytical effect estimates are predominantly based on selfreported estimates of sitting time, which are associated with substantial measurement error. Studies with accurate 
measurement of sitting posture, such as the one by van der Berg et al [1], are therefore most welcome - especially if they can also be extended to a longitudinal design, to minimise the risk of reverse causality (i.e. when individuals are already on the disease pathway and therefore display or report more sitting). To explore whether reverse causality affected the results of their cross-sectional study, the authors ran a sensitivity analysis in which they excluded participants with type 2 diabetes on insulin medication (who were assumed to have more severe diabetes). This did not change the overall results. However, a major limitation of cross-sectional research is the lack of inference on the temporal sequence between exposure (e.g. sedentary time) and outcome (e.g. type 2 diabetes), which will inherently always be associated with substantial risk of reverse causality. Another common limitation in this research area is residual confounding for poorly or unmeasured confounders. This is when a relationship is estimated incorrectly because a confounding factor was not measured (such as dietary intake, as acknowledged by the authors) or not sufficiently accounted for (such as moderate-to-vigorous physical activity, as described further below).

These difficulties hamper our current understanding of the exact impact prolonged sitting may have on the development of chronic disease, such as type 2 diabetes. Excessive sedentary time is, however, ubiquitous in most modern societies, both at an inter- (i.e. in the majority of individuals across all age groups) and intra-individual level (i.e. on average more than half of the waking day) [7]. This means that even if true effect estimates are relatively small, the potential population impact of any behavioural change in terms of prevention of type 2 diabetes and other chronic disease may still be relevant.

\section{Opportunities to improve the evidence base}

\section{Patterns of sedentary time: optimising its operationalisation in observational research Unlike other health behaviours, for} example smoking, where even minimal exposure such as passive smoking may increase risk, not all sitting is harmful. Indeed, some sitting is needed for rest and recovery, and prolonged time spent in non-sedentary postures (e.g. static standing) also has inherent risks. Attention has therefore shifted to also considering, and intervening on the patterns of sedentary time, i.e. how sedentary time is accrued.

Definitions of sedentary patterns vary between observational studies. This is testament to the relatively recent interest in this behavioural construct as a potential independent health hazard. Healy et al [8] introduced this concept by relating the number of 'breaks' or interruptions in sedentary time to specific cardiometabolic risk factors. This breaks construct, which has been incorporated into public health messages, is now widely used in studies looking into accumulation patterns of sedentary time. However, this frequency measure may not be the most robust estimate of patterns of sitting time, partially because it does not directly assess duration of prolonged sitting bouts per se [9]. Other indicators have therefore also been considered, some of which are also frequency-based (e.g. number of prolonged bouts), whereas others are more duration-based (e.g. average sedentary bout duration). As also reported by van der Berg et al [1], these different constructs show associations of different strengths, which indicates that they may not relate equally strongly to the same latent construct of 'prolonged sitting'. This diversity may complicate comparisons between study results and general inference about the importance of sitting patterns as regards health. More work is therefore needed in terms of optimising and harmonising pattern definitions for observational research. Constructs that are conceptually more similar to assessing prolonged sitting per se may need to be used more universally, such as time spent in bouts of minimal durations (e.g. $\geq 30 \mathrm{~min}$ ). These constructs can also be easily translated into public health guidelines (e.g. 'Get up at least every $30 \mathrm{~min}$ ') if they were to be associated with increased health hazard. Concurrently, more studies should implement posturediscriminating methods that can accurately distinguish sitting from standing, and therefore also identify the transitions between these postures.

Integrating the use of improved measures of sitting time, higher intensity activities and domain-specific information As mentioned above, the study by van der Berg et al [1] is to be commended for the use of posture-discriminating accelerometry. This is currently rare on such a scale; the vast majority of previous studies have implemented traditional hip- or waist-mounted accelerometers [10]. Despite this, however, time spent in moderate-to-vigorous physical activity, an important co-variate, was measured in a more rudimentary manner. This problem, common in this field of research, could be overcome in future research by combining different objective inference methods, each specialised in accurately capturing specific subcomponents of the intensity or posture-based spectrum of activities. Further important opportunities involve the integrated use of simultaneously measured domain-specific information (e.g. through domain-specific logs [11] or GPS), ensuring participant burden remains low. This would allow an understanding of the context of the behaviour and associated opportunities for intervention.

Considering the $\mathbf{2 4} \mathrm{h}$ clock Notably, activities do not occur in isolation, but, rather, are interdependent. That is, given a $24 \mathrm{~h}$ day, time spent doing one activity necessarily displaces time spent doing another. The impact of sedentary time is therefore not only due to the time spent in a low energy, low muscle contractile state, but also due to the loss of time spent in higher intensity activities. An individual's health is impacted by the relative balance between sleep, sedentary behaviour and physical activity. More advanced analytical techniques, 
such as iso-temporal substitution modelling [12] and compositional data analysis [13], have recently been introduced in sedentary behaviour/physical activity epidemiology. These approaches aim to account for this interdependency of activity behaviours in aetiological analyses with health outcomes. They therefore provide more realistic insights into the relative impact of a behavioural change from one part of the intensity spectrum to another, using observational data. As expected, these models have typically shown that shifting time from sedentary to moderate- or vigorous-intensity activities generally has the greatest benefit on markers of cardiometabolic health [13]. However, given the challenges associated with increasing population levels of moderate- to vigorousintensity activity, it is encouraging to note that some cardiometabolic benefits may be achieved with shifts from sedentary to light-intensity activities, including standing [14]. An important consideration in the use of these techniques is the assurance that they provide us with insights that are informative and easily translatable into public health guidance.

\section{Establishing robust evidence in a prospective and global} setting The van der Berg et al study [1], and the vast majority of other observational studies that have related objectively measured sedentary time with health outcomes, have been crosssectional. This is because implementation of these activity monitors in larger aetiological studies is a more recent development, especially those monitors which are able to accurately discriminate between postures [10]. Future longitudinal assessment in cohorts such as that in the Maastricht study [1] will reduce the potential risk of findings being affected by reverse causality. As effect estimates for some of these associations may also be fairly small, future data sharing between multiple cohorts will facilitate an increase in statistical power. This provides additional opportunities in terms of understanding differences in dose-response associations within and between populations. Given the predominance of non-posture-discriminating methodologies in ongoing cohorts, this may also encourage endeavours to harmonise data between different methodologies [10].

In terms of experimental research, several laboratory-based physiological trials have indicated the potential acute benefits of breaking up prolonged sitting, especially in terms of limiting postprandial glucose and insulin excursions (for example, see [15]). Future acute physiological trials, with further control for the frequency, duration and intensity of breaks, will advance our understanding of the physiological underpinnings of observational findings. Alongside these laboratorybased studies, ongoing free-living intervention research is providing evidence for the feasibility of changing prolonged sitting behaviour [16]. These studies now need to be followed by trials that are sufficiently powered to allow assessment of the longer term effects of changes in free-living sitting on cardiometabolic and other health outcomes. These trials should also control for compensatory effects in terms of sitting behaviour and total energy expenditure.

\section{Conclusion}

We applaud the study by van der Berg et al [1], which provides important new insights into this area of research. Robust findings from future longitudinal observational and experimental studies, which engage with the wealth of current and future opportunities (as described above), are now needed. These will place us in a better position to (1) make more realistic inferences about the public health impact of changing activity intensity levels in a balanced, efficient and realistic manner; (2) subsequently shape more refined public health guidance in line with this evidence; and, last but not least, (3) translate these insights into individual, micro- and macro-level societal changes, which reduce the public health burden associated with the distorted balance of activity intensity levels, as currently witnessed around the world.

Funding KW is supported by a British Heart Foundation Intermediate Basic Science Research Fellowship (grant number FS/12/58/29709) and the UK Medical Research Council (grant number MC_UU_12015/3). GNH is supported by an Australian National Health and Medical Research Council Career Development Fellowship (grant number 108029).

Duality of interest The authors declare that there is no duality of interest associated with this manuscript.

Contribution statement Both authors were responsible for drafting the article and revising it critically for important intellectual content. Both authors approved the version to be published.

\section{References}

1. van der Berg JD, Stehouwer CDA, Bosma H et al (2016) Associations of total amount and patterns of sedentary behaviour with type 2 diabetes and the metabolic syndrome: The Maastricht Study. Diabetologia. doi:10.1007/s00125-015-3861-8

2. Sedentary Behaviour Research Network (2012) Standardized use of the terms "sedentary" and "sedentary behaviours". Appl Physiol Nutr Metab 37:540-542

3. Hu FB, Leitzmann MF, Stampfer MJ, Colditz GA, Willett WC, Rimm EB (2001) Physical activity and television watching in relation to risk for type 2 diabetes mellitus in men. Arch Intern Med 161:1542-1548

4. Hu FB, Li TY, Colditz GA, Willett WC, Manson JE (2003) Television watching and other sedentary behaviors in relation to risk of obesity and type 2 diabetes mellitus in women. JAMA 289:1785-1791

5. Wilmot EG, Edwardson CL, Achana FA et al (2012) Sedentary time in adults and the association with diabetes, cardiovascular disease and death: systematic review and meta-analysis. Diabetologia 55: 2895-2905

6. Department of Health, Physical Activity, Health Improvement and Protection (2011) Start active, stay active. A report on physical activity 
for health from the four home countries' Chief Medical Officers. Available from: www.dh.gov.uk/en/Publicationsandstatistics/ Publications/PublicationsPolicyAndGuidance/DH_128209

7. Aresu M, Becares L, Brage S, et al (2009) Health survey for England 2008, volume 1: physical activity and fitness. The NHS Information Centre for Health and Social Care, Leeds

8. Healy GN, Dunstan DW, Salmon J et al (2008) Breaks in sedentary time: beneficial associations with metabolic risk. Diabetes Care 31: 661-666

9. Chastin SF, Egerton T, Leask C, Stamatakis E (2015) Meta-analysis of the relationship between breaks in sedentary behavior and cardiometabolic health. Obesity 23:1800-1810

10. Wijndaele K, Westgate K, Stephens SK et al (2015) Utilization and harmonization of adult accelerometry data: review and expert consensus. Med Sci Sports Exerc 47:2129-2139

11. Wijndaele K, De Bourdeaudhuij I, Godino JG, Lynch BM, Griffin SJ, Westgate K, Brage S (2013) Reliability and validity of a domain-specific last-7-day sedentary time questionnaire. Med Sci Sports Exerc 46:1248-1260
12. Mekary RA, Willett WC, Hu FB, Ding EL (2009) Isotemporal substitution paradigm for physical activity epidemiology and weight change. Am J Epidemiol 170:519-527

13. Chastin SF, Palarea-Albaladejo J, Dontje ML, Skelton DA (2015) Combined effects of time spent in physical activity, sedentary behaviors and sleep on obesity and cardio-metabolic health markers: a novel compositional data analysis approach. PLoS One 10: e0139984

14. Healy GN, Winkler EA, Owen N, Anuradha S, Dunstan DW (2015) Replacing sitting time with standing or stepping: associations with cardio-metabolic risk biomarkers. Eur Heart J 36:2643-2649

15. Dunstan DW, Kingwell BA, Larsen R et al (2012) Breaking up prolonged sitting reduces postprandial glucose and insulin responses. Diabetes Care 35:976-983

16. Healy GN, Eakin EG, Lamontagne AD et al (2013) Reducing sitting time in office workers: short-term efficacy of a multicomponent intervention. Prev Med 57:43-48 